SCIENCE CHINA

Physics, Mechanics \& Astronomy

\title{
High-pressure opens new windows for topological materials
}

\author{
HongMing Weng ${ }^{1,2 *}$ \\ ${ }^{1}$ Beijing National Laboratory for Condensed Matter Physics, Institute of Physics, Chinese Academy of Sciences, Beijing 100190, China; \\ ${ }^{2}$ Collaborative Innovation Center of Quantum Matter, Beijing 100190, China
}

Received October 30, 2017; accepted November 1, 2017; published online December 18, 2017

Citation: H. M. Weng, High-pressure opens new windows for topological materials, Sci. China-Phys. Mech. Astron. 61, 038231 (2018), https://doi.org/10.1007/ s11433-017-9129-0

Recently Guo et al. [1] have performed a systematic simulation on the high-pressure phases of the first experimentally available Weyl semimetals TaAs family. Through such a cheap but powerful technique, they theoretically found a new topological phase in this family members under high pressure. This article presents a nice example that high-pressure is applicable in tuning the topological phase transition.

Though Weyl fermions still remain elusive to be discovered as a new type of particle, their analogues have been proposed as emergent quasiparticles in condensed matter systems [2]. Recently, the existence of Weyl semimetal has been predicted [3] and experimentally confirmed in TaAs family compounds. The characteristic surface Fermi arcs [4] and negative magnetoresistance $[5,6]$ due to Weyl nodes have been observed. On the other hand, high pressure is an effective toolkit to modify the crystal structure, electronic structures, and expectedly the topological properties of materials. In fact, squeezing the materials slightly will enhance the band width and alternate the on-site energy of the related orbitals, in which way the band topology is to be changed as schematically demonstrated in Figure 1 where the genus of donut is changed. While some high-pressure phases of TaAs [7,8] and $\mathrm{TaP}[9]$ have been studied, the high-pressure phase diagrams of $\mathrm{NbP}$ and $\mathrm{NbAs}$ are still unknown. A systematic study on the high-pressure phase diagrams for whole family ( $\mathrm{NbP}, \mathrm{NbAs}, \mathrm{TaP}$, and TaAs) is expected for more complete and systematical exploration of them.

Guo et al. [1] studied the high-pressure phases of the

*Corresponding author (email: hmweng@iphy.ac.cn) whole TaAs family systematically up to $100 \mathrm{GPa}$. Using crystal structure searching techniques in combination with first-principles calculations, they managed to predict the most stable structures at different pressures. Their method had been successful in finding stable high-pressure phases of many systems, including TaAs [7] and TaP [9]. The theoretical results here show that the sequence of the high-pressure phase transitions of TaAs and $\mathrm{NbAs}[10]$ are very similar $\left(I 4_{1} m d \rightarrow P \overline{6} m 2 \rightarrow P 2_{1} / \mathrm{c} \rightarrow P m \overline{3} m\right)$, although the transition pressures are different. NbP [10] and TaP are slightly different from their arsenide, where new structures appear, namely the $\mathrm{Cmcm}$ phase in $\mathrm{NbP}$ and the Pmmn phase in TaP, respectively.

Notably, the $P \overline{6} m 2$ phase NbAs is a new topological semimetal, similar to the $P \overline{6} \mathrm{~m} 2$-TaAs. There are two types of intriguing fermions coexisting in this phase, including Weyl fermions and triply degenerate fermions. Its topological

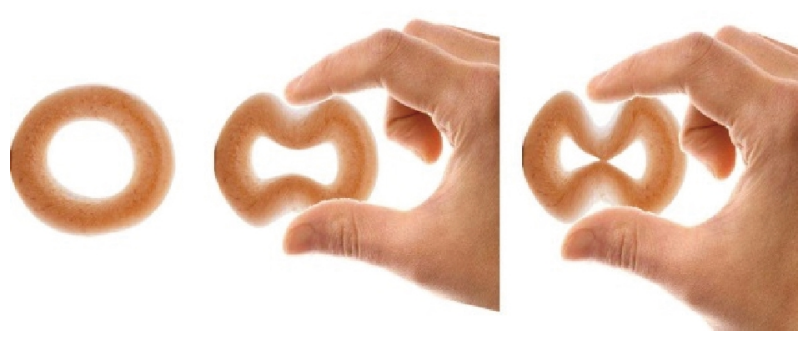

Figure 1 (Color online) Squeezing a donut with topological invariant genus number of 1 drives it to a new one with genus number 2, which is a topological phase transition. 
properties are indicated by the non-trivial surface states. Another important point is that, all the Weyl points in this $P \overline{6} \mathrm{~m} 2$ phase are at the same energy level, sharing the same shape and travelling at the same Fermi velocity. This is very different to its ambient $I 4_{1}$ md phase, which has two inequivalent Weyl cones at different energy level.

This article shows that pressure is a powerful tool to tune crystal structures and the topological properties of materials. It would stimulate further high-pressure experimental investigations, not only on the TaAs family, but also on other topological materials.

This work was supported by the National Key Research and Development Program of China (Grant No. 2016YFA0300600), the National Natural Science Foundation of China (Grant Nos. 11674369, and 11422428), and the "Strategic Priority Research Program (B)" of the Chinese Academy of Sciences (Grant No. XDB07020100). The author thanks Wei Wang for the figure plotting.

1 Z. Guo, P. Lu, T. Chen, J. Wu, J. Sun, and D. Xing, Sci. China-Phys. Mech. Astron. 61, 038211 (2017)
2 X. Wan, A. M. Turner, A. Vishwanath, and S. Y. Savrasov, Phys. Rev. B 83, 205101 (2011), arXiv: 1007.0016.

3 H. Weng, C. Fang, Z. Fang, B. A. Bernevig, and X. Dai, Phys. Rev. X 5, 011029 (2015), arXiv: 1501.00060.

4 B. Q. Lv, H. M. Weng, B. B. Fu, X. P. Wang, H. Miao, J. Ma, P. Richard, X. C. Huang, L. X. Zhao, G. F. Chen, Z. Fang, X. Dai, T. Qian, and H. Ding, Phys. Rev. X 5, 031013 (2015), arXiv: 1502.04684

5 X. Huang, L. Zhao, Y. Long, P. Wang, D. Chen, Z. Yang, H. Liang, M. Xue, H. Weng, Z. Fang, X. Dai, and G. Chen, Phys. Rev. X 5, 031023 (2015), arXiv: 1503.01304.

6 J. H. Du, H. D. Wang, Q. Chen, Q. H. Mao, R. Khan, B. J. Xu, Y. X. Zhou, Y. N. Zhang, J. H. Yang, B. Chen, C. M. Feng, and M. H. Fang, Sci. China-Phys. Mech. Astron. 59, 657406 (2016), arXiv: 1507.05246

7 Y. Zhou, P. Lu, Y. Du, X. Zhu, G. Zhang, R. Zhang, D. Shao, X. Chen, X. Wang, M. Tian, J. Sun, X. Wan, Z. Yang, W. Yang, Y. Zhang, and D. Xing, Phys. Rev. Lett. 117, 146402 (2016).

8 H. Wang, H. Wang, Y. Chen, J. Luo, Z. Yuan, J. Liu, Y. Wang, S. Jia, X. J. Liu, J. Wei, and J. Wang, Sci. Bull. 62, 425 (2017).

9 Y. Li, Y. Zhou, Z. Guo, X. Chen, P. Lu, X. Wang, C. An, Y. Zhou, J. Xing, G. Du, X. Zhu, H. Yang, J. Sun, Z. Yang, Y. Zhang, and H.-H. Wen, arXiv: 1611.02548.

10 J. Zhang, C. Yang, W. Rao, J. Hao, and Y. Li, Sci. Rep. 7, 13251 (2017). 